\title{
Evolutionary side-steps
}

\author{
Ronald Sluys \\ Expert-center for Taxonomic Identification, Institute of Taxonomic Zoology, University of Amsterdam, \\ P.O. Box 4766, 1009 AT Amsterdam, The Netherlands
}

Keywords: ontogeny, larvae, evolutionary theory, parallelism, homology

Review of: Larvae and evolution - Toward a new zoology. By D.I. Williamson. Chapman \& Hall, New York/ London, 1992. xvi +223 pp. ISBN 0-412-03081-0. US $\$ 39.95$.

Many of the lower metazoans reach adulthood via complex developmental stages. Each of these stages is described with a specialized terminology, which is different for every major group of organisms. Biologists with a proper training in biodiversity may have learned about these complex issues during their undergraduate days, but many of us do no longer encounter the intricacies of lower metazoan development in later stages of our careers. We may be familiar with the ontogenetic stages in the group on which we specialize, but only a few will have practical knowledge and an overview of the larval stages in such groups as crustaceans, echinoderms, polychaetes, sipunculans, molluscs, flatworms, bryozans, and nemertines. Donald Williamson certainly belongs to the last-mentioned category of biologists.

In this book, Williamson addresses the situation that marine invertebrates from highly distinct and not closely related phylogenetic lines may develop from very similar larvae. There are many examples of distinct lower metazoan lineages sharing highly similar early developmental stages.

Decapod crustaceans partly are classified into the Brachyura (or true crabs) and the Anomura. Confusingly, some "crabs", like the hermit crabs, are not true crabs or brachyurans but are anomurans. The larvae of brachyurans and anomurans are totally different. However, brachyuran crabs from the superfamily Dromioidea have larvae that do not look at all like proper brachyuran larvae but have a very close resemblance to larvae of the Anomura. Furthermore, it is known that there are animals that superficially look like crabs but are no true crabs at all, being members of the Anomura. Well known examples are the anomuran king crabs (family Lithodidae) for which it has been argued recently that they form the sister group of a particular lineage of hermit crabs (Cunningham et al., 1992). In view of this, Williamson asks the question whether the dromioid crabs are true brachyurans (despite their anomuran larvae; and how to explain this?), or do they represent another example of anomurans that underwent carcinization (evolved a crablike appearance, as in the king crabs).

Adult echinoderms (starfishes, brittle-stars, sea-urchins, sea-cucumbers, sea-lilies, and the sea-daisy Xyloplax) generally are radially symmetrical animals, constructed along five radial elements, or multiples of five. The various types of echinoderm larvae, however, are bilaterally symmetrical. Williamson asks, How could such a unique discrepancy between larvae and adults evolve?

Hemichordates (Enteropneusta or acorn worms, and Pterobranchia) look completely different from echinoderms, at least as adults. Enteropneust larvae, however, show a striking resemblance to those of echinoderms. Metamorphosis starting with these similar larvae is totally different in enteropneusts and echinoderms. How did such a situation arise in the course of evolution?

Sea-urchins (Class Echinoidea) and brittle-stars (class Ophiuroidea) are very different as adults but develop from a very similar and unique type of larva, the pluteus. How did that come about?

Trochophore larvae are characteristic of such diverse phyla as the Annelida, Echiura, Sipuncula, and Mollusca. If one rejects convergence or parallelism as well as evolutionary conservatism (i.e. inheritance from a remote common ancestor) of the larvae as causal explana- 
tions, as Williamson does, then an alternative explanation is called for. How then to explain the anomaly of adult diversity in these groups and their larval uniformity?

Polyclad flatworms, nemertines, and bryozoans at one stage during their metamorphosis all have a trochophorelike larva, a situation that cannot be accounted for easily by conventional evolutionary theory, according to "Larvae and evolution".

In several chapters, Williamson outlines the dissimilar adult stages and the similar larval stages in the groups of taxa mentioned above. In each chapter he argues that the observed facts cannot be accounted for by conventional explanations, and instead he sketches for each case an evolutionary scenario of ancestors and descendants that fits his preferred and newly proposed hypothesis.

This new hypothesis proposes that embryonic and larval forms have occasionally been transferred between distinct evolutionary lineages, i.e., result from horizontal (lateral) larval or genetic transfer. According to this hypothesis “... larvae of sponge crabs (Dromioidea) resemble those of hermit crabs (Paguroidea) ... because an ancestor of all modern sponge crabs acquired a larva from a hermit crab" (p. 3). And "the larvae of echinoderms resemble ... those of acorn worms (Enteropneusta) not because of common ancestry but because echinoderms had no planktonic larvae until one of them acquired larvae from an acorn worm. Derivatives of this larval form were then acquired successively by ancestors of all the echinoderms that today have larvae. Among these derivatives are the so-called pluteus larvae of brittle-stars and sea-urchins ... [The] similarity between larvae of these two classes of echinoderms is evidence not of common ancestry but of larval transfer from a seaurchin to a brittle-star long after the adult groups were established"' (p. 3).

In point of fact, Williamson proposes two hypotheses, one being the occurrence of larval or genetic transfer and the second that such transfers have resulted from crossfertilizations. Chapter 13 discusses some possible tests of the larval transfer hypothesis and outlines the preliminary results of cross-fertilization experiments. Successful crosses between species from different superfamilies or so are considered to lend strong support for both the larval transfer hypothesis and its presumed mechanism, cross-fertilization.

In reading this book, systematists will have no difficulty in recognizing that it deals with fascinating cases of parallelism. The book concentrates on larvae, but the phenomenon is not confined to larvae or to the groups discussed by Williamson. There are many other cases where the same character re-appears in rather distantly related taxa, while it is absent in other, more closely related groups. I wrote a paper about the implications of rampant parallelism for phylogenetic analysis (Sluys, 1989), illustrating the reality of the phenomenon with examples from flatworms. In that paper, I attempted also to give a causal explanation for rampant parallelism: retention of structural gene complexes (but no expression in particular taxa) and subsequent re-expression (in other, distantly related taxa) due to a change in regulatory mechanisms. On p. 35-36 Williamson mentions precisely this mechanism as a possible causal explanation for the distribution of his larvae over the phylogenetic tree. It is one of the very few places in the book where he realizes that more explanations/mechanisms are possible then solely his preferred one.

That Williamson does not present other possible explanations for parallelism in larvae probably stems from his less than up to date view about modern evolutionary theory. It is obvious from numerous places in the text that for Williamson evolutionary theory started and ended with Darwin, nothing really new has been added since. Furthermore, all biologists agree that evolution is "Darwinian" and proceeds by "series of small changes" under the influence of natural selection, according to the writer. If Williamson cannot find any intermediate stages, or cannot imagine any then he assumes something strange and unexplainable under the Darwinian paradigm must have taken place (see, for example, p. 67). It happens quite often that Williamson cannot imagine a particular evolutionary scenario and that ipso facto evolution cannot have followed that path. For example, on p. 117 he states that it is "difficult to envisage how they [different types of metamorphoses in trochophores] could have evolved from one ancestral method purely by species-to-species descent with modification'. After some discussion of the particular situation he then concludes (p. 119) that neo-Darwinian theory seems to have no satisfactory explanation for the different types of metamorphoses in different groups. Also on p. 148 he argues that a new type of nervous system could not have evolved gradually while the old system continued to function. This argumentation is used to support the idea of larval transfer. However again, it is based on a preconceived and restricted idea about what can happen during evolution. The evolutionary history of the secondary hinge of the lower jaw in mammals clearly shows that the old (reptilian) and the newly evolved joint existed and were functional in mammal-like reptiles for millions of years (Romer \& Parsons, 1977). Modern evolutionary theory can come up with a number of feasible explanations; it is much more flexible and comprehensive than 
granted by Williamson. In short: Williamson uses a too restrictive and outdated idea about present evolutionary theory. It may serve the author to make his point, but it is hardly a balanced presentation and evaluation of his view against other feasible ones.

A few other examples from the book can illustrate the author's concept of evolutionary theory and evolutionary processes. The situation is described (p. 60-65) that the similar larvae of enteropneusts and echinoderms after the initial stages show a highly different metamorphosis, although they evolved from a common ancestor. This is considered to be something requiring a special explanation, which cannot be offered by current theories. However, this case appears to me as an example of a situation that one encounters often and can be accounted for by current evolutionary theory. Every group of organisms, after having diverged from a common ancestor, may acquire any amount of unique characteristics (autapomorphies), in larval as well as in adult stages. It depends on the tempo of (morphological) evolution in that particular group. There is no clock that prescribes that so many features newly evolve per so many million years - we do not know beforehand. Birds and crocodiles accumulated a great number of differences after they diverged from their common ancestor; nevertheless they are each other's closest relatives among living organisms.

A similar case is described on p. 116, where it is concluded that the trochophore larvae of polychaetes, echiurians, sipunculids, and molluscs are very similar. They agree so much that the conventional explanation, postulating that they did not change for millions of years, does not hold true, according to Williamson. I think he exaggerates this case, apparently to hammer his point home. First, there is no reason why these larvae must diverge because such happens in other groups of animals (Williamson's argument on top of p. 116). Second, the similarity between trochophore larvae is exaggerated: between and within the groups discussed there certainly are differences in larval characters. According to SalviniPlawen (1988) the trochophores of Mollusca, Echiura, and Polychaeta are rather different; Ivanova-Kazas (1985) concludes that there are differences but that they are all trochophores sensu lato. Therefore, one cannot say that these larvae have been "immune" (p. 116) to evolutionary change. Williamson admits (p. 117) that there is a small amount of variation between the trochophores of the various phyla. According to him, however, these differences can be explained only by his theory that a larval form has spread relatively recently over the phyla, otherwise they would have had much more time to diverge. But how does one know beforehand how fast evolution goes in particular cases? There is no law requiring that after so much time so much morphological diversification must have been accumulated.

On p. 86-87 Williamson admits not to be able to imagine an evolutionary process whereby sea-urchins and brittle-stars would have acquired their similar pluteus larvae through convergence (he means in this case: as a result of necessary adaptation to the same environment). He even states that the situation is "inconsistent" (p. 87) with a convergence hypothesis. However, again, does not Williamson give nature (and present evolutionary theory) too little credit? There are many cases wherein very similar structures appear to have evolved in very different groups. Furthermore, Williamson does not provide adequate arguments for the homology of Ophioplutei and Echinoplutei. To the contrary: "ophioplutei never have preoral arms" (p. 82), "some echinoplutei, but no ophioplutei have muscles that can be used to vary the angles between the longer arms on the opposing sides" ( $p$. 83). Williamson allows for limited convergence between echinopluteus and ophiopluteus larvae (p. 86), but his main question is whether echinopluteus and ophiopluteus could have reached their broadly similar form as a result of convergence (p. 86). (His answer is no, it came about through horizontal gene transfer.) But "broadly similar form" does not strike me as a particularly interesting scientific phenomenon, requiring a special explanation. Replace echinopluteus and ophiopluteus larvae by dolphins and ichthyosaurs and the superficiality of the problem becomes obvious. What we need are comparisons between homologous features; and homology is not a concept to be passed over easily.

Poor application of the concept of homology can be found throughout the book. This disturbs me because as long as it has not been shown or made plausible that structures are essentially the same, it is of no use inventing evolutionary explanations or scenarios for their evolution. On p. 131-134 it is said that "The affinity of polyclad larvae to trochophores has long been accepted and is confirmed by the general similarity of the nervous system to that of a larval polychaete ...". "General similarity" does not convince me easily!

"Larvae and evolution" provides the reader with rather elaborate descriptions of adult morphology of the groups under consideration. Although initially this reads quite well, it eventually detracts from the real issue. In view of the above, I would have preferred more detailed discussions on the morphology (and especially ultrastructure) of the various larvae. This would have enabled proper assessments of homology, going beyond present statements that particular larvae are quite similar. 
In many places in the book the terms "relatedness", "related", and "affinity" are used. It is never very clear what is meant by them. Frequently I got the impression that all Williamson means in such cases is similarity. This probably results from the fact that the book does not put the problem in a clear phylogenetic perspective, thus unequivocally separating superficial similarity and genealogical relationship. This is evident, for example, from the usage of only one "phylogenetic" tree, viz. the one published by Barnes in 1980. Barnes' tree is certainly not a phylogenetic tree in the modern sense of the word; furthermore, it is mostly an unresolved bush and it is based on an authoritarian and outdated methodology. Why has not Williamson taken a couple of modern phylogenetic trees (based on phylogenetic principles) and used these for making his comparisons? This is very important because the topology of the phylogenetic tree tells us whether particular cases of alleged parallelism or convergence concern closely related taxa or distantly related taxa. Williamson apparently tries to defend his position on p. 17-20 where he informs the reader that systematists still quarrel about how to construct a proper phylogenetic tree and classification, and that "there is no general consensus among biologists as to the principles which should be employed in classifying organisms or in deducing phylogenetic trees" (p. 19). Examination of recent systematic literature quickly reveals that the quarrel is now over and that there is almost universal agreement that the phylogenetic, Hennigian procedure is the best method available at the moment for constructing phylogenetic trees. With respect to classifications, opinions still differ, but for evolutionary biology this does not matter because it is phylogenies we are interested in, not classification per se. On p. 19 Williamson also seems to defend his position with the statement that his book has nothing to do with views on classification or phylogeny (reconstruction), it is not the object of the book. Definitely, it is precisely the object of the book, or should be. It is only through a proper phylogenetic tree that Williamson's observations are put into perspective; without any phylogenetic tree (thus also excluding the one of Barnes, 1980) we would not even recognize the problem!

Therefore, Williamson should have made use of modern phylogenetic trees on the origin and evolution of the Metazoa (cf. several papers in Fernholm et al., 1989; Brusca \& Brusca, 1990; Meglitsch \& Schram, 1991). I shall illustrate my point on proper phylogenetic trees by taking one of the cases discussed in the book. On p. 108 it is stated that it is "generally assumed" that Echinodermata and Hemichordata are offshoots of the same major branch of the phylogenetic tree. First, "generally as- sumed" is too vague a statement; who assumes that and based on what sort of analysis? Second, the statement is much too imprecise: what exactly is the genealogical relationship between Echinodermata and Hemichordata? According to some modern phylogenetic trees these taxa stand relatively wide apart (cf. Brusca \& Brusca, 1990). Furthermore, it is said that "it has been argued that the similar larvae reflect the true affinities of the adults" ( $p$. 108). Who says so? I cannot find this in recent literature. It looks as if Williamson creates here an alleged problem on the basis of old and vague arguments. He proposes, of course, his theory of larval transfer in this case, but properly analysed phylogeny allows for another interpretation: Brusca \& Brusca (1990) postulate trochophore larvae as a synapomorphy for the monophyletic group [Sipuncula, Echiura, Mollusca, Annelida, Pogonophora, Vestimentifera, Arthropoda] under the assumption of secondary loss in the Arthropoda.

There are two other weaknesses in the book that relate to the lack of adequate phylogenetic reasoning: (1) storytelling/inventing scenarios, (2) apparent lack of awareness of systematic literature discussing ontogenetic sequences and their relation to phylogenetics. In the absence of a phylogenetic tree based on detailed research, one is not constrained in sketching evolutionary scenarios; anything goes and there is no way of testing such stories. Nevertheless, Williamson maintains that his evolutionary scenarios to explain embryological discrepancies are "more probable" (p. 73) than other, more traditional hypotheses. But his "more probable" is based solely on story-telling. Without the constraints of a phylogenetic tree/hypothesis (which informs you about evolutionary pathways of your features; the tree itself is based on other characters) I can think of an infinite number of scenarios and then claim that these are "more probable".

I give one more example of what I consider to be storytelling: chapter 9 on "Echinoderms: fossil record". The entire chapter is based on story-telling with a lot of "it is suggested", "it seems not unlikely", "it seems probable", "it may be suggested that", etc. etc. In the absence of adequate fossils and/or phylogenetic data, an innumerable number of scenarios can be invented, among which one that fits precisely the horizontal gene transfer hypothesis. Although the author admits (p. 106) that the story is "largely conjectural" and that additional data "are far from sufficient to prove the theories now being advanced", I think it is a weak chapter.

In the phylogenetic/cladistic literature, there is an ongoing discussion about ontogeny and phylogeny and how we may use one to illuminate the other. Up to 1988 this 
discussion has been summarized in the book "Ontogeny and systematics" (Humphries, 1988). I think this discussion has great relevance for the topic discussed by Williamson, but I have not found any indication that he is aware of this ongoing scientific debate, which is still far from any consensus. It has at least become clear that due to dedifferentiation, paedomorphosis, insertion and deletion (terminal and non-terminal) of developmental stages it can be extremely difficult (sometimes impossible) to deduce the genealogical hierarchy from ontogenetic transformation series.

I do agree with Williamson that an explanatory, historical hypothesis can be correct without us knowing the causal, driving mechanism of the phenomena. My favorite example is the hypothesis of continental drift which was postulated many years before we knew anything about plate tectonic mechanisms. And I think he is also right in exploring possible mechanisms and to try to "avoid confusing cause and effect" (p. 160). This is what Williamson tries to do in chapter 13 ("Toward confirmation of the hypotheses'), but I cannot say that he succeeds very well, which is particularly due to a peculiar philosophy of science. I think it is now generally accepted that one cannot confirm or prove hypotheses, one can only test, falsify, or corroborate hypotheses. On p. 161 Williamson claims that his hypotheses are consistent with all observations and that therefore they provide the best explanations. However, from a philosophical point of view no one deliberately erects an hypothesis that is discordant with the facts to be explained, thus, concordance with the facts is not a measure of truth.

On p. 160 a parallel is drawn between extinction of dinosaurs and the theory of larval transfer, thus illustrating the point about avoiding confusion about cause and effect in historical biology. The reasoning is invalid. That dinosaurs once existed and are now extinct is merely observation of a pattern in biodiversity. This cannot be equated with horizontal gene transfer because the latter cannot be observed in a similar way, i.e. it is not a pattern but a process. What we do observe are all sorts of parallelisms that may be the result of various kinds of processes, among which horizontal gene transfer (others are modifications of early development, and reexpression). It is not necessary to know at present on which mechanism horizontal gene transfer is based, but it remains a process of explanation. On p. 192 it is written that "The present evidence that hybridization has taken place during the course of evolution comes from the distribution of types of early development in the animal kingdom, but, if this evidence is accepted, could not the same process have produced ..." (italics mine). Again, there is no question of evidence or process in the distribution of developmental types; it is the observation of a pattern in nature.

The first half of chapter 13 further sketches, in a simplistic manner, a number of alleged tests of larval transfers. For example, on p. 162-163 Williamson discusses the situation that chemo-immunotaxonomy and gel electrophoresis will be able to assess the "relatedness" (genealogy?) between various groups of organisms. However, it has been known for some time now that these methods are very inadequate for this purpose. Gel electrophoresis of enzymes might mean something on a very low taxonomic level, but it is certainly incapable of showing whether organisms belong to the same genus or family. These days one relies on much more rigorous molecular methods, although it is certain also that these are not the panacea for resolving phylogenetic relationships. And why would biochemical or molecular methods come up with something better than morphological (but still phylogenetic) procedures anyway? (and such is implied by Williamson).

The second half of chapter 13 discusses the author's cross-breeding experiments, which are designed to test ( $p$. 169), to confirm his theory. In view of the rather preliminary results, obtained only recently, and the absence of good documentation, these experiments do not provide the fascinating support for the theory one would hope for. The author reports to have produced blastulae by fertilizing ascidian eggs with sea-urchin sperm which in some cases developed into pluteus larvae. However, no details are given about methods and experimental design and papers on these experiments have not yet been published. Furthermore, Williamson fails to provide high quality photographs of crucial stages and observations, e.g. of a ciliated blastula hatching from an ascidian egg.

On p. 198 it is concluded that the evidence for his theory is considerable and that several tests have been suggested. However, I conclude that (1) no hard evidence has been presented (at best particular patterns in character distribution), (2) the case presented suffers from problems of method, and (3) that the section on proposed tests comes from one of the weakest parts of the book.

Lynn Margulis has successfully championed the idea that early in their history protoctists, Fungi, plants, and animals evolved from bacterial ancestors by a series of symbioses (Margulis, 1981). The possibility of gene transfer has attracted mostly the attention of microbiologists and botanists, who may postulate microbial parasites as possible vectors of gene transfer (cf. Heron, 1992). Lynn Margulis and Alfred I. Tauber wrote a foreword to 
"Larvae and evolution" in order to pave the way for reading Williamson's ideas and testing his theory. Margulis and Tauber realize that the ideas expressed are idiosyncratic and that the experimental evidence presented does not match the exceptional and far-stretching claims made in the book, but they argue that Williamson deserves to be heard. It is clear from the foreword that they fear that scientists are not prepared to consider seriously and objectively the concept of larval transfer. But for myself, I have no difficulty examining the merits of a working hypothesis on horizontal gene and larval transfer. It is not the principle/hypothesis of horizontal larval and gene transfer that worries me, but it is the way in which Williamson argues his case.

\section{References}

Barnes, R.D., 1980. Invertebrate zoology (4th ed.): 1-1089 (Saunders, Philadelphia).

Brusca, R.C. \& G.J. Brusca, 1990. Invertebrates: i-xviii, 1-922 (Sinauer, Massachusetts).
Cunningham, C.W., N.W. Blackstone \& L.W. Buss, 1992. Evolution of king crabs from hermit crab ancestors. Nature, 355: 539-542.

Fernholm, B., K. Bremer \& H. Jörnvall (eds.), 1989. The hierarchy of life: molecules and morphology in phylogenetic analysis: 1-499 (Excerpta Medica, Amsterdam).

Heron, C., 1992. The networks of botanical creation. New Scientist, 8 Feb. 1992 (1807): 40-44.

Humphries, C.J. (ed.), 1988. Ontogeny and systematics: $i-x i$, 1-236 (Columbia Univ. Press, New York).

Ivanova-Kazas, O.M., 1985. Origin and phylogenetic significance of trochophore larvae. Zool. Zh., 64: 485-497.

Margulis, L., 1981. Symbiosis in cell evolution: i-xxii, 1-419 (W.H. Freeman \& Comp., San Francisco).

Meglitsch, P.A. \& F.R. Schram, 1991. Invertebrate zoology (3rd ed.): 1-623 (Oxford University Press, Oxford).

Romer, A.S. \& T.S. Parsons, 1977. The vertebrate body (5th ed.): i-viii, 1-624 (Saunders, Philadelphia).

Salvini-Plawen, L. von, 1988. Annelida and Mollusca - a prospectus. Mikrofauna marina, 4: 383-396.

Sluys, R., 1989. Rampant parallelism: an appraisal of the use of nonuniversal derived character states in phylogenetic reconstruction. Syst. Zool., 38: 350-370.

Received: 9 September 1992 